\title{
Relationship between Symptoms in Women with Endometriosis and Lifestyles: a Qualitative Interview Study
}

\author{
Scavone M. ${ }^{{ }^{*}}$, Gizzi C. ${ }^{1}$, Albi E. ${ }^{1}$
}

\begin{abstract}
Endometriosis is a common condition that affects reproductive-aged women and is characterized by the presence of endometrial tissue outside the uterine cavity with nodules and lesions. The study aimed to analyze lifestyles of women affected by endometriosis in relation with their symptoms. In this observational study, 735 Italian women have been interviewed online through a questionnaire structured in two parts. The first part was mainly anamnestic, focused on gathering information about the age, the stage of disease, comorbidities, involved organs, symptomatology, chirurgical treatment, induced menopause. The second part focused on the individual characteristics and lifestyle of the patients such as the body mass index, physical activity, quality of sleep, and the diet. The results showed how a healthy diet and a regular physical activity reduce drastically the symptoms of the patients.
\end{abstract}

Keywords: endometriosis, diet, physical activity, body mass index, quality of sleep

${ }^{1}$ Department of Pharmaceutical Science, University of Perugia, Perugia, Italy

${ }^{*}$ Corresponding author: Albi, E.

*E-Mail: elisabetta.albi@unipg.it

DOI: $10.2478 /$ ebtj-2020-0022

() 2020 Authors. This work was licensed under the Creative Commons AttributionNonCommercial-NoDerivs 4.0 License.

\section{Introduction}

Endometriosis is a worldwide common gynecological condition that affects women in reproductive age. The pathology is characterized by the endometrium growth at the outside of its physiological localization, such as the myometrium, ovary, and peritoneum with consequent inflammation, fibrosis, and formation of adhesions (1). The disease can be asymptomatic or it can manifest with pelvic pain during menstrual bleeding and/or sexual intercourse, by influencing the quality of life (2). In addition, it represents the major cause of infertility (3). The exact pathogenetic mechanism has not been established but the most accepted hypotheses go from the retrograde menstruation and genetic or immunologically linked susceptibility (4) to Müllerian rests (5) or exposition to chemical sources that alter the endocrine system (6). The retrograde menstruation is characterized by an outflow of the endometrial lining through the fallopian tubes. This retrograde flow, together with the potential hematogenous or lymphatic circulation, can result in the seeding of endometrial tissue into ectopic sites $(7,8)$. Although most women have retrograde menstruation, not all of them have endometriosis. The immune dysfunction in the patients interferes with the elimination of the lesions themselves (9). Endometriosis is also associated with an increased risk of autoimmune diseases and endometrioid ovarian cancer as well as other cancers, including lymphoma and non-Hodgkin's melanoma (9). As above reported, endometriotic lesions can also result from Müllerian remains that have not undergone differentiated or failed to migrate properly during fetal development $(10,11)$. Of note, Müllerian anomalies may be the cause of the obstruction of menstrual outflow (12). Furthermore, also somatic mutations resulting in growth dysregulation can be considered 
etiological factors $(9,10)$. Additional etiological factors may be prolonged exposure to endogenous estrogen such as due to early menarche, late menopause or obesity (12). The treatment can be pharmacological or surgical but the correct lifestyle is also relevant (13). Of considerable importance are the quality of sleep, sports activity and diet. In fact, the low physical activity and the poor sleep quality are associated with the endometriosis symptoms (14). Particular attention was payed to the diet, by indicating that endometriosis is associated with a low intake of vegetable, fruit (15), and vitamin D (16), and with a high trans-fatty acid (17), beef and other kinds of red meat and alcohol (18) consumption. This is relevant thanking in account that exists a relationship between lipids and vitamin D (19). On the other hand, the use of omega-3 fatty acids, antioxidants and a combination of vitamins and minerals positively influences the endometriosis-associated symptoms (20), considering that lipids are involved in immunity and inflammatory processes $(21,22)$. There are currently no studies that establish a simultaneous correlation of the sleep quality, sports activity and diet with the symptoms of endometriosis. The present work is a qualitative interview study aimed to highlight in the same patients different aspects of endometriosis in the Italian population.

\section{Materials and Methods}

\section{Patients}

735 women affected by endometriosis we included in the study realized between February 4th to March 4th 2020. The exclusion criteria were obesity and cancer. The patients were recruited online on the Facebook page of National Association Endometriosis Project (APE-ODV) and participated to the study on a voluntary basis by giving their informed consent.

\section{Interview}

A specific questionnaire was prepared and administered online. The questionnaire was structured in two parts. The first part was mainly anamnestic, focused on gathering information about the age, the stage of disease, comorbidities, involved organs, symptomatology, chirurgical treatment, induced menopause. The second part focused on the individual characteristics and lifestyle of the patients such as the body mass index, physical activity, quality of sleep, and the diet.

\section{Results}

\section{Population characteristics}

The analysis of the interview indicated that the average age of the 735 women who agreed to participate in the study was $36.5+17.5$ years. In particular, $5 \%$ aged $18-25,37 \%$ aged $26-35$, $48 \%$ aged $36-45$, and $10 \%$ over the age of 46 . From the results was evident that $85 \%$ aged $26-45$. Interestingly, the mean age at the time of diagnosis was $28+4$ years. It is important to highlight that $84 \%$ of patients had received the diagnosis more than 1 year after the onset of symptoms and only $2 \%$ within 1 year, by indicating that often the endometriosis diagnosis is tardive. The remaining 14\% refers to women that have been diagnosed in few months. This result justifies the stage of the disease at the time of diagnosis, reported by the patients. In fact, $47 \%$ of women examined were already at IV stage of the disease, $12 \%$ at III stage, $6 \%$ at II stage, $6 \%$ at I stage, and $29 \%$ of patients didn't remember the stage they belonged to. Only $18 \%$ of patients did not present comorbidities. Figure 1 shows the percentage of different pathologies of $72 \%$ of women with comorbidity. A data that caught particularly the attention is the high percentage of women with autoimmune pathologies which covers $6 \%$ of the examined women of whom $62 \%$ were affected by celiac disease (Fig.1).

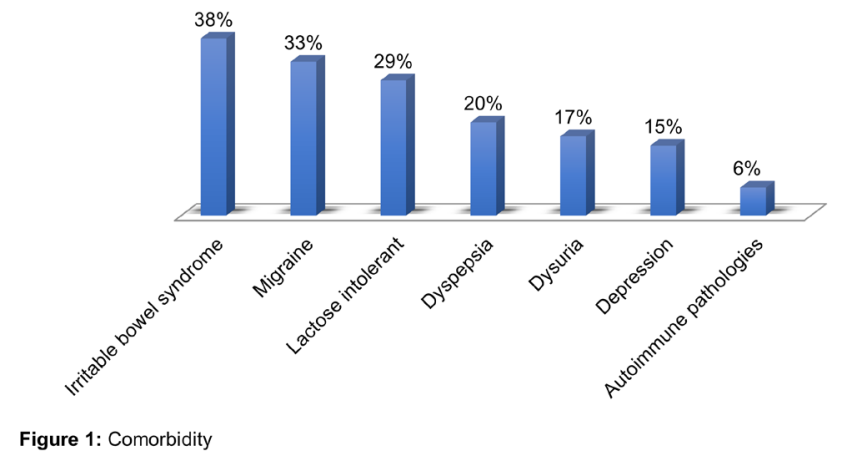

The presence of comorbidities is another factor that sometimes makes early diagnosis difficult.

\section{Clinical picture}

From the interview it was clearly evident that several organs were involved in the endometriosis, many of these were simultaneously present in the same patient. In figure 2, the percentage of involved organs.

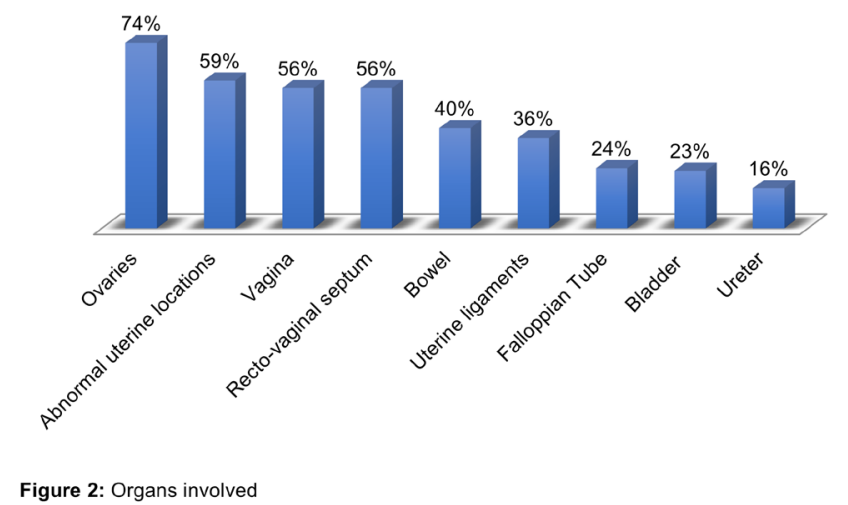

As a consequence, the symptomatology linked to endometriosis is wide and complex. The main reported symptom at the moment of the diagnosis was the dysmenorrhea (Fig.3). 


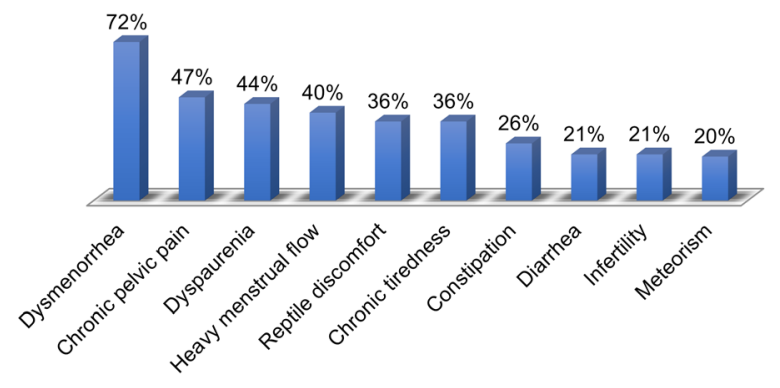

Figure 3. Symptomatology at the moment of the diagnosis

Unfortunately, the symptom that leads to the diagnosis of endometriosis is not strictly related to this symptom. In fact, almost half of women had a chronic pelvic pain (47\%), dyspaurenia (44\%) and heavy menstrual flow (40\%). These are symptoms until today underestimated, that caracterises the majoruty of women affected by endometriosis. A discrete percentage of women affected by this disease showed reptile discomfort (36\%), interpreted as false sensation of needing to defecate, rectal pain, discomfort to stay normally seated, sense of posterior heaviness. In addition, chronic tiredness is often neglected, even though it is a frequent symptom; $36 \%$ of women examined showed this symptom. Moreover, $26 \%$ of patients presented constipation, $21 \%$ diarrhea, $21 \%$ meteorism and flatulence phenomenon. Only $6 \%$ of patients were asymptomatic.

Very relevant were the symptomatology in relation with menstrual cycle and in the fertility. 56\% of women indicated symptoms away from the menstrual cycle, $31 \%$ symptoms during the menstrual cycle and right before it and, 13\% symptoms only during the menstrual cycle. Often women find out to be affected by endometriosis only after a few failed attempts to conceive, followed by further investigations and studies that confirmed the incapability to conceive. According to this, from the 735 women, only $30 \%$ managed to conceive of whom $62 \%$ had a disease at the III o IV stage. As consequence, $44 \%$ of women were in menopause. Of these, $84 \%$ were on a pharmacological menopause, $10 \%$ are on a surgical menopause and, $6 \%$ are on a spontaneous menopause. Considering that $78 \%$ of patients had undergone surgery, either laparoscopic or with laparotomy, it is evident that when patients arrived for surgery, many were already in pharmacological or spontaneous menopause. Among all women that had a surgical treatment, 36\% faced a bowel resection or rectal shaving. Among the women examined, $62 \%$ followed a pharmacological therapy and of these $78 \%$ suffered from collateral effects. Many effects were simultaneously present in multiple patients. Therefore, $43 \%$ presented abnormal vaginal discharge, $30 \%$ increase in appetite, $28 \%$ vaginal candidiasis or other fungal infections, 55\% headache, $44 \%$ hot flashes, $76 \%$ decreased libido, $13 \%$ hypertrichosis, $72 \%$ body weight changes, $25 \%$ nausea, $8 \%$ diarrhea, $33 \%$ hair loss, $63 \%$ nervousness, $60 \%$ depression.

\section{Lifestyles}

BMI

BMI is an important factor for the health of subject and its value is calculated considering height and weight. Generally, in adult, BMI between 18.50 and 24.99 is considered as normal-weight, BMI between 16.00-18.49 as under-weight, BMI between 25.00 and 29.99 as over-weight and, BMI above 30 as obesity. As above reported, the patients with obesity were not included in the study. Of the 735 women, $68 \%$ were normal weight, $22 \%$ under-weight, and $10 \%$ over-weight. On a closer look the youngest women had more adequate BMI than older women. More specifically $69 \%$ of women over 36 were overweight. These data are relevant considering that the high BMI is strictly related with inflammation of the adipose tissue and an inflammatory picture is not a positive factor for endometriosis.

\section{Physical activity}

From this study it has been observed how the physical activity is beneficial on those who work out on a regular basis. However, only $17 \%$ of women examined worked out and reported positive results on their health conditions. In details, 33\% of women did not work out and another important percentage (30\%) worked out only when had time (Fig. 4).

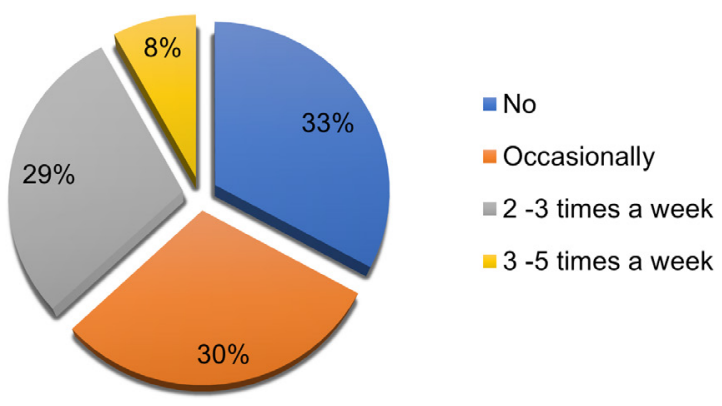

Figure 4. Physycal activity frequency

It has been observed how women over 46 years showed more interest in working out. It resulted evident that women with the age between 18 and 25 years and women over 45 did not like physical activity. In fact only $7 \%$ of women over 45 and $2 \%$ of women with the age between 18 and 25 years practiced physical activity. The majority of women confirmed that used walk for long distances. Among the sport most performed it has been mentioned: yoga, pilates, jogging, swimming e water gym. It is obvious the importance of a healthy and correct lifestyle in order to prevent some pathologies.

\section{Quality of sleep}

$21 \%$ had a bad quality of sleep, $46 \%$ of women reported a discreet quality of sleep, $28 \%$ had a good quality of sleep, and only $5 \%$ had an excellent quality of sleep. Interestingly, by compar- 
ing the chronic tiredness, as one of the symptoms in women affected by endometriosis, with the quality of sleep it appeared evident that among $36 \%$ of women that suffered from chronic fatigue syndrome, $76 \%$ suffered from poor- quality of sleep; in particular $27 \%$ of patients suffered from a very bad quality of sleep, while $49 \%$ suffered from discreet quality of sleep; only $23 \%$ had a good quality of sleep and a smaller percentage had an excellent quality of sleep.

\section{Diet}

$75 \%$ of the examined women thought that nutrition plays a key role on the reduction of the pain linked to the inflammation induced by endometriosis. $46 \%$ followed a healthy diet and in particular women aged from 36 to 45 years old. There are several nutritional regimes that can be associated to different ethical, environmental, religious choices and often influenced by current trends. (Fig. 5). According to this aspect, it has been observed that $77 \%$ of examined women followed an omnivorous diet, $9 \%$ follows a vegetarian diet, $2 \%$ follows a vegan diet, about $12 \%$ followed alternative diets (ketogenic, macrobiotic, dukan ect.).

- Omnivorous diet $\square$ Vegetarian diet $\square$ Vegan diet $₫$ Others

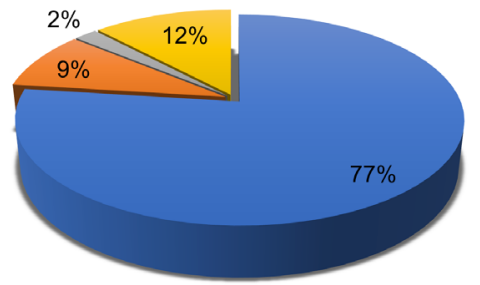

Figure 5. Nutritional Regimes

In the study we showed how milk and milk derivatives exacerbated the most painful condition (on 59\% of patients) followed by sugar, refined flour and baked goods form it derived (on $50 \%$ of patients), by indicating that these nutrients might facilitate inflammation process (Fig. 6). Notably, 35\% of patients ingested these nutrients 2-4 times a week. In the figure 6 it has been reported the consumption of each type of food during the week

It is known that additional foods that contribute to pain conditions in endometriosis patients are soy and red meat. From the analysis of the responses it was highlighted that women had been alerted about this. In fact, most women did not consume soy and consumed red meat once a week (Fig. 7).

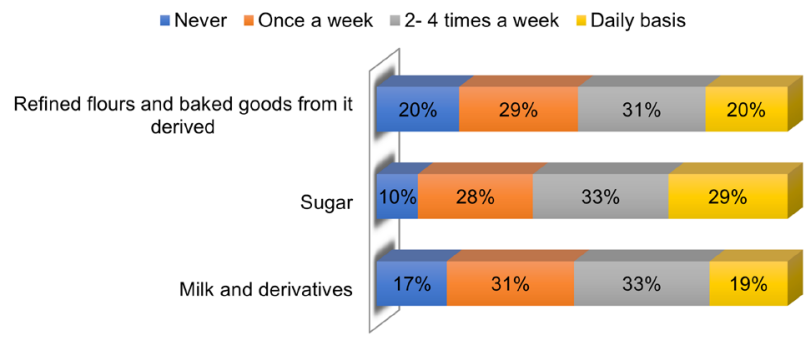

Figure 6. Main nutrients that induce pain

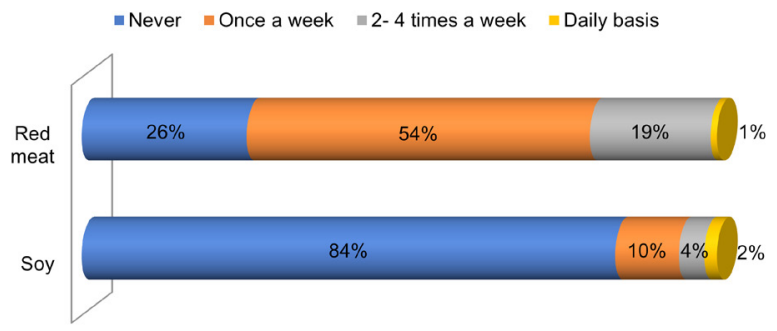

Figure 7. Soy and read meat consumption

Additionally, cured and processed meats (40\% of cases), alcohol (35\% of cases), coffee, tea and drinks with caffeine (29\% of cases), pre-cooked and readymade goods (28\% of cases), chocolate ( $26 \%$ of cases), legumes (17\% of cases) increased the pain. Only in some cases fruits (7\%) and vegetables (10\%) increased the pain condition. It is important to know how cooked vegetables, on $58 \%$ of cases, provoked more discomfort compared to raw vegetables. It is really difficult to establish exactly which food is responsible for a pain because several foods often cause pain in the same patient. Patients were well educated about the importance of water consumption. Despite this, $40 \%$ of women consumed 1 liter or less of water per day. The most relevant symptoms consequent to the consumption of the above nutrients included abdominal bloating (88\%), pelvic pain (40\%) and chronic tiredness (34\%) (Fig. 8).

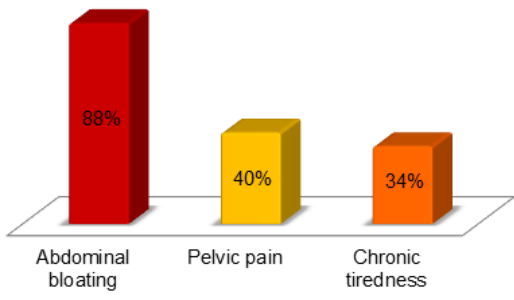

Abdominal bloating

$\square$ Pelvic pain

Chronic tiredness

Figure 8. Symptoms associated to pro-inflammatory food consumption 
Some symptoms are less frequent, but still relevant such as diarrhea, constipation, migraine, dysuria, nausea, meteorism and dyspepsia.

\section{Discussion}

Considering how nowadays lifestyle influences the health of the single person, this study aims to prove the association between lifestyle of a specific group of women who share a common disease. The results obtained in the present study support the difficulty in making an early diagnosis due to the variability of the tissues and organs presented and the comorbidities. The symptoms of the disease are so constant, even if variable, that they become part of the patient's life. It is difficult to determine whether lifestyle is one of the causes of pain or whether pain determines lifestyle. It is likely that this is a vicious cycle. Therefore, it is essential to know all the factors that can block this cycle and a correct and continuous information on physical activity and nutrition is necessary. Besides the usual approaches, from a couple of years it has been suggested to follow a personalized nutrition regime in order to support the individual medical treatment. These nutritional regimes are mainly focused on the importance of supporting the caloric needs of the patient and potential preferences towards certain food, avoiding the consumption of the so-called pro-inflammatory goods. A correct nutrition status is essential in order to reduce the symptomatology related to endometriosis. There are many foods identified by patients as the cause of increased pain. Therefore, it will be important to carry out a specific food plan in the individual subject and also in relation to the menstrual cycle. Red meat and soy are less ingested probably because different scientific studies reported how these two particular foods might cause the endometriosis disease. It has been demonstrated that a specific natural integrator based on lipoic acid, palmitoiletanolamide and myrrh reduces the pelvic pain in endometriosis (23). Moreover, very useful are omega-3 and omega- 6 fatty acids (24) that play a role as antioxidant molecules (25). Moreover, the study has highlighted that the average age to conceive tends to rise. This can be related on one hand to socio-economical problems that affect couples nowadays, on the other hand to the pathology that often requires the intervention of assisted reproduction techniques. Therefore, an early diagnosis may be beneficial in terms of quality of life and in terms of fertility preservation.

\section{Conclusions}

In conclusion this study indicates how even for more complex pathologies like endometriosis, a regular physical activity and a correct nutritional strategy associated to the pharmacotherapy and surgical-therapy, visibly can improve the pain conditions of the patients and her lifestyle.

\section{Conflicts of Interest}

The authors declare no conflict of interest.

\section{References}

1. Brown J, Crawford TJ, Datta S, Prentice A, and Cochrane Gynaecology and Fertility Group. Cochrane Database Syst Rev. Oral contraceptives for pain associated with endometriosis. Cochrane Database Syst Rev. 2018(5): CD001019. doi: 10.1002/14651858.CD001019.pub3.

2. Moradi M, Parker M, Sneddon A, Lopez V, Ellwood D. Impact of endometriosis on women's lives: a qualitative study. BMC Womens Health. 2014;14:123. doi: 10.1186/14726874-14-123

3. Jacobson TZ, Barlow DH, Koninkx PR, Olive D, Farquhar C. Laparoscopic surgery for subfertility associated with endometriosis. Cochrane Database of Syst Rev 2002 (4). doi: 10.1002/14651858.CD001300

4. Crosignani P, Olive D, Bergqvist A, Luciano A. Advances in the management of endometriosis: an update for clinicians. Hum Reprod Update. 2006; 12(2):179-89. doi: 10.1093/humupd/dmi049.

5. Montgomery GW, Mortlock S, Giudice LC. Should Genetics Now Be Considered the Pre-eminent Etiologic Factor in Endometriosis? J Minim Invasive Gynecol. 2020;27(2):280-286. doi: 10.1016/j.jmig.2019.10.020. E

6. Diamanti-Kandarakis E, Bourguignon J P, Giudice LC, Hauser R, Prins GS, Soto AM, Zoeller RT, Gore AC. Endocrine-disrupting chemicals: an Endocrine Society scientific statement. Endocr Rev. 2009;30:293- 342. DOI: 10.1210/er.2009-0002

7. Farland LV, Shah DK, Kvaskoff M, Zondervan K, Missmer SA. Fattori di rischio epidemiologici e clinici per endometriosi. In: D’Hooghe T, editore. Biomarcatori per endometriosi. Springer Science; New York: 2015

8. Berkley KJ, Rapkin AJ, Papka RE. I dolori dell'endometriosi. Scienza. 2005; 308: 1587-9

9. Giudice LC. Clinical practice. Endometriosis. N Engl J Med. 2010 Jun 24; 362

10. Bulun SE. Endometriosis. N Engl J Med. 2009; 360:368-79

11. Sampson JA. Endometriosi metastatica o embolica dovuta alla diffusione mestruale del tessuto endometriale nella circolazione venosa. Am J Pathol. 1927; 3: 93-109

12. Missmer SA, Hankinson SE, Spiegel-man D, Barbieri RL, Marshall LM, Hunter DJ. Incidenza di endometriosi laparoscopicamente confermata da fattori demografici, antropometrici e di stile di vita. Am J Epidemiol. 2004; 160: 784-9 60

13. Zito G, Luppi L, Giolo E, Martinelli M, Venturin I, Di Lorenzo G, Ricci G. Medical treatments for endometriosis-associated pelvic pain. Biomed Res Int 2014;2014:191967 doi: 10.1155/2014/191967.

14. Youseflu S, Jahanian Sadatmahalleh S, Roshanzadeh G, Mottaghi A, Kazemnejad A, Moini A. Effects of endometriosis on sleep quality of women: does life style factor make a difference? BMC Womens Health. 2020;20(1):168. doi: 10.1186/s12905-020-01036-z.

15. Fjerbaek A, Knudsen UB. Endometriosis, dysmenorrhea and diet--what is the evidence? Eur J Obstet Gynecol Reprod Biol. 2007;132(2):140-7. doi: 10.1016/j. 
ejogrb.2006.12.006.

16. Lerchbaum E, Rabe T. Vitamin D and female fertility. Curr Opin Obstet Gynecol. 2014;26(3):145-50. doi: 10.1097/ GCO.0000000000000065.

17. Hansen SO, Knudsen UB. Endometriosis, dysmenorrhoea and diet. Eur J Obstet Gynecol Reprod Biol. 2013;169(2):162-71. doi: 10.1016/j.ejogrb.2013.03.028.

18. Jurkiewicz-Przondziono J, Lemm M, Kwiatkowska-Pamuła A, Ziółko E, Wójtowicz MK. Influence of diet on the risk of developing endometriosis. Ginekol Pol. 2017;88(2):96102. doi: 10.5603/GP.a2017.0017.

19. Patria FF, Ceccarini MR, Codini M, Conte C, Perioli L, Beccari T, Albi E. A Role for Neutral Sphingomyelinase in Wound Healing Induced by Keratinocyte Proliferation upon 1a, 25-Dihydroxyvitamin D3 Treatment. Int J Mol Sci. 2019 Jul 25;20(15):3634. doi: 10.3390/ijms20153634.

20. Huijs E, Nap A. The effects of nutrients on symptoms in women with endometriosis: a systematic review.Reprod Biomed Online. 2020;41(2):317-328. doi: 10.1016/j. rbmo.2020.04.014.

21. Caso V, Parnetti L, Panarelli P, Magni MPV, Gallai V, Albi E. Selection of thrombogenetic antiphospholipid antibodies in cerebrovascular disease patients. J Neurol 2003 May;250(5):593-7. doi: 10.1007/s00415-003-1045-9.

22. Albi E, Alessenko A, Grösch S. Sphingolipids in inflammation. Mediators of inflammation 2018;2018:7464702. doi: $10.1155 / 2018 / 7464702$

23. De Leo V, Cagnacci A, Cappelli V, Biasioli A, Leonardi D, Seracchioli R. Role of a natural integrator based on lipoic acid, palmitoiletanolamide and myrrh in the treatment of chronic pelvic pain and endometriosis. Minerva Ginecol 2019; 71:191- 5. DOI: 10.23736/S0026-4784.19.04384-3

24. Gazvani MR, Smith L, Haggarty P, Fowler PA, Templeton AA. High omega-3:omega- 6 fatty acid ratios in culture medium reduce endometrial-cell survival in combined endometrial gland and stromal cell cultures from women with and without endometriosis. Fertil Steril 2001;76(4):71722. doi: 10.1016/s0015-0282(01)01991-4.

25. Codini M, Tringaniello C, Cossignani L, Boccuto A, Mirarchi A, Cerquiglini L, Troiani S, Verducci G, Patria FF, Conte C, Cataldi S, Ceccarini MR, Paroni R, Dei Cas M, Beccari T, Curcio F, Albi E. Relationship between Fatty Acids Composition/Antioxidant Potential of Breast Milk and Maternal Diet: Comparison with Infant Formulas. Molecules. 2020;25(12):2910. doi: 10.3390/molecules25122910. 